\title{
Evaluation of Container Terminal Arrangement By Computer Simulation
}

\author{
Takeshi Shinoda ${ }^{1}$, Hideyo Inutsuka ${ }^{2}$, Putu Hangga ${ }^{3}$ \\ and Tiago Novaes Mathias ${ }^{4}$
}

\begin{abstract}
With growing ship size and demand for effective handling of container cargoes in recent years, automation has inevitably took place in almost all aspect of modern terminal operation. So far, automation has been led by the large terminals in Europe and North America and soon it will become a pressure for terminals in developing countries to go for automation. The drawbacks heavily rely on the initial investment cost and question arises with regard to effectiveness of automation for medium and small size container terminals. A visual environment that simulates container terminal operation is developed to facilitate investigation advantage and disadvantages of automated container terminal compared to conventional container terminal. A discrete event model for container handling process and agent-based model for path mover flow system is built and validated based on operating terminal in real world and visually simulated to serve the objective. The result of the computer simulation is an evaluation of performance metric for both terminal concepts under similar arrangements. In addition, visual simulation was able to notify the area where advantage and disadvantage of both concepts will take place during operation.
\end{abstract}

Keywords - container terminal, automation, computer simulation, visual simulation environment, performance metric, performance evaluation

\section{INTRODUCTION}

$\mathrm{T}$ he decision to increase ship size by major shipping lines leads to a higher requirement for container terminal. Container terminals have responded to lower growth by investing in handling equipment and IT infrastructure to accommodate larger ships, which pose challenges and require adaptations. Having limited land sources for container stacks and the lack capability of horizontal transport, maintaining productivity will require dramatic innovation in the handling systems or operational methods and has opened a way for automation to be introduced in recent years [1]. It makes sense for individual players but not for other facing softer annual throughput. Huge investment needs to be made to replace older asset and a lot of complexity have to be taken into consideration to accommodate change or increase terminal capacity.

The main driver for the introduction of automation is to reduce the cost per handled container in the terminal. Other key deciding factors to introduce automation would be reliability, predictability and safety of operations and reduced environmental impact [2]. The advent of automation is focused on unmanned container handling equipment process automation in the form of Terminal Operating System (TOS) integration with the information flow from ships, internal and external transportation. While the soft infrastructure of automation can be relatively easier to be adopted, hard infrastructure of the automated terminal might requires a

\footnotetext{
1,3 Department of Marine Systems Engineering, Kyushu University, Fukuoka 819-0395, Japan.

Corresponding Email: shinoda@nams.kyushu-u.ac.jp

2,4 Graduate School of Engineering, Department of Urban and Environmental Engineering, Kyushu University, Fukuoka 819-0395, Japan.
}

major and significant change to container terminal layout. An automated container terminal with unmanned equipment mostly use either Automated Guided Vehicle (AGV), Automated Straddle Carrier (A-STRAD) or Shuttle Carrier as their in-yard carrier system and Automated Stacking Crane (ASC) for yard operation. Conventional yard layout that is parallel to the quay is considered to be outdated and inefficient, and perpendicular to the quay layout has been preferable to minimize the total distance of horizontal transport with heavy burden to the two operating ASCs in each container block.

Most of automated container terminals with perpendicular layout in the world are either located in a strategic hub for an international container trade or a newly built container terminal as an expansion from existing terminal with high annual throughput. On the other hand, layout change is not an easy case for medium and small size container terminals with an annual throughput less than 2 million TEUs. The authors believe that most of container terminal in the world will fall under this category, especially the one with fairly average geostrategic advantage and located in a developing country. This dilemma has been one of the main obstacles to the adoption of automation technology. There are problem of port design and adoption of technology that requires deep understanding on how the current system can work out to cope with challenges and increase its performance with less influence of the physical impact of the automation to terminal layout. An assessment method will be needed to fairly evaluate whether automated container terminal with perpendicular layout is better than conventional terminal with parallel layout.

To address this issue, the paper is constructed in twofold; initiate a benchmarking approach to fairly evaluate performance metrics of layout orientation (parallel or perpendicular to the quayside) using its default arrangements and investigate the performance by simulation model based on existing terminal setup. The 
second section of this paper will briefly explain the layout and arrangement of parallel and perpendicular

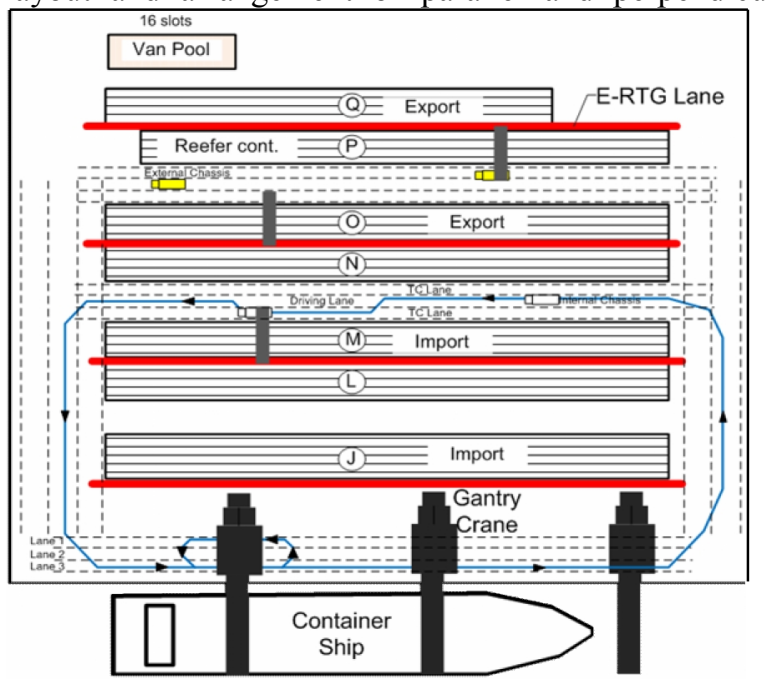

(a) Existing parallel layout of ICCT layout as well as introducing performance metrics that needs to be evaluated.

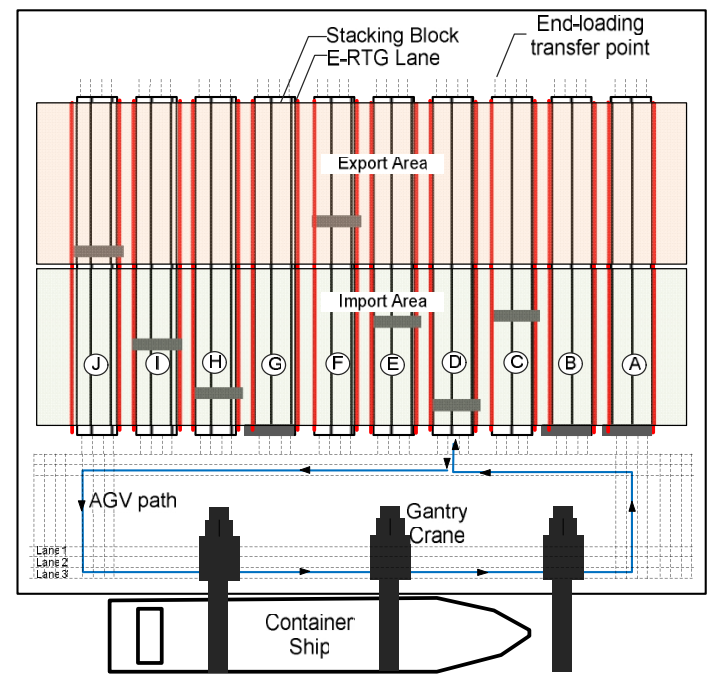

(b) Conceptual perpendicular layout

Figure 1. Layout concepts under evaluation

The third section deals with conceptual model of the container terminals and simulation setup. The fourth section will discuss the result of the simulation and compare the performance metrics based on the proposed benchmark.

\section{CONTAINER TERMINAL ARRANGEMENT}

Much of the theoretical literature on port planning and performance evaluation are detached from the existing environment with regard to design arrangements, handling systems, operating procedures and technology variations [3]. Therefore, this paper will use existing container terminal as the basis of examination to relate theory and port operating practice and show evident regarding to the usability of our approach.

Japan is widely known for automation in its vehicle manufacture industry. However, automation is less utilized for container terminals in Japan. Tobishima Container Terminal in Nagoya City is the only automated container terminal in Japan so far and it still use parallel terminal layout. Perpendicular layout for automated terminal is less preferable mainly because of high initial investment and complex adjustment required for layout modification. Both automated and nonautomated terminals utilize similar vertical transport system; Gantry crane for seaside operation and mainly Rubber-Tired Gantry, either diesel powered (RTG), automated (ASC) or Electrified (E-RTG) for container stacking in the yard. For simplification of terms, yard cranes for both systems will be defined as Transfer Crane (TC) in this paper. The major difference of both layouts will be the means of horizontal transport system and layout orientation and transfer points locations of storage yard as shown in Figure 1.

\section{A. Parallel Layout of Container Terminal}

The terminal arrangements explained in this section use the terminal layout and cargo handling equipment arrangement of Island City Container Terminal (ICCT) in Fukuoka City - Japan. The yard arrangements in ICCT consist of truck-chassis wheeled system as carrier system for horizontal transport within the terminal. The storage yard operation is based on sharing policy of RTG which allows RTG to move around different yard blocks without restriction. Since ICCT utilize E-RTG type of TC for yard operation, there is electric tray with steel structure system bolted on top of a concrete base at one side of each container block. Electrical energy is picked up from the conductor rails using a collector trolley connected to the TC. Therefore, only one TC is usually placed in one container block at a time due to restriction of electric consumption of the terminal. The container stacking sequences in the yard is usually pre-determined by scheduler in a way that TC movement along the block is minimized.

\section{B. Perpendicular Layout of Container Terminal}

The concept of automated container terminal investigated in this paper is based on EUROMAX container terminal layout where orientations of stacks are arranged perpendicular to the quay. We brought the layout concept and stack orientation and implement it in accordance to the basic terminal dimension of ICCT. The horizontal transport of containers between the quay and the storage yard is carried out by Automated Guided Vehicle (AGV). AGV are similar to conventional truckschassis system but operate on a pre-defined guide path without drivers. Each block in the container storage yard is occupied with at least two ASC type of TC serving at both end of the block for import-export containers (seaside operation) and receipt-delivery containers (gate operation). As opposed to the parallel layout, the number and location of yard transfer points (TP) is significantly different. There are 4 TP placed at every end of each blocks. Due to the smaller of TP attached to a block and the orientation of the block significant increase of total covered distance of TCs is expected to happen. 


\section{Benchmarking Operational Performance}

The goal of every container terminal is to perform efficiently and maintain competitiveness by providing low cost and high quality services to customers. In import container operation case, the container ship's berthing time has to be minimized. In other word, designated GCs have to operate at the maximum productivity during the work shift [4]. TABLE 1 .

Performance metrics to evaluate parallel and perpendicular terminal layout

\begin{tabular}{|c|c|c|c|}
\hline No & Performance metrics & Definition of metrics & Measured dimensions \\
\hline \multirow[t]{3}{*}{1} & \multicolumn{3}{|l|}{ Gantry crane (GC) metrics } \\
\hline & a. Productivity & Number of handled container by GC. & (1) Unit (Box/hour/GC) \\
\hline & b. Waiting time & Time spent by GC to wait for carrier systems (CS) arrival. & (2) Unit (sec/box) \\
\hline \multirow[t]{7}{*}{2} & \multicolumn{3}{|c|}{ Carrier systems (CS) (PM) metrics } \\
\hline & a. Working time & $\begin{array}{l}\text { Time required for carrier systems (CS) from picking up } \\
\text { container from GC and travel until reach yard transfer point } \\
\text { (TP). This measurement is not including the time carrier } \\
\text { systems (CS) spent when travels in empty condition. }\end{array}$ & $\begin{array}{l}\text { (1) Total (sec) } \\
\text { (2) Average (sec/pm), } \\
\text { (3) Unit (sec/box) }\end{array}$ \\
\hline & b. Moving time & $\begin{array}{l}\text { Time where the carrier systems (CS) are moving during } \\
\text { simulation. Measured in total (hour) and average } \\
\text { (hour/carrier systems (CS)) }\end{array}$ & $\begin{array}{l}\text { (1) Total (hour) } \\
\text { (2) Average (hr/pm) }\end{array}$ \\
\hline & c. Covered distance & $\begin{array}{l}\text { Distance covered by carrier systems (CS) during } \\
\text { simulation. }\end{array}$ & $\begin{array}{l}\text { (1) Total }(\mathrm{km}) \\
\text { (2) Average }(\mathrm{km} / \mathrm{pm})\end{array}$ \\
\hline & d. Idle time & $\begin{array}{l}\text { Idle time of carrier systems (CS) is parked and waiting for } \\
\text { a task to be conducted. }\end{array}$ & $\begin{array}{l}\text { (1) Total (sec) } \\
\text { (2) Average (sec/pm) }\end{array}$ \\
\hline & e. Wait time to transfer & $\begin{array}{l}\text { Time spent by carrier systems (CS) waiting to transfer } \\
\text { container to TC. Measured in Total (sec), average } \\
\text { (sec/carrier systems (CS)) and unit (sec/carrier systems } \\
\text { (CS)/box) }\end{array}$ & $\begin{array}{l}\text { (1) Total (sec) } \\
\text { (2) Average }(\mathrm{sec} / \mathrm{pm}) \text {, } \\
\text { (3) Unit }(\mathrm{sec} / \mathrm{pm} / \mathrm{box})\end{array}$ \\
\hline & e. Time in motion & $\begin{array}{l}\text { Total percentage of situation where } \mathrm{TC} \text { is in a motion } \\
\text { during the simulation run. }\end{array}$ & (1) $\%$ \\
\hline
\end{tabular}

3 Transfer crane (TC) metrics
a. Working time
Time required for TC from picking up container from carrier systems (CS) and stack container to designated location. This measurement not including TC retrieval time
(1) Total (sec)
(2) Average (sec/TC) on empty condition.
b. Covered distance
Total horizontal distance covered by TC during simulation
(1) Total (m)
(2) Average $(\mathrm{m} / \mathrm{TC})$,
(3) Unit (m/TC/box)
c. Time in motion
Total percentage of situation where TC is in a motion
(1) $\%$ during the simulation run.

\section{Overall handling metrics}
a. Service time
Handling time of container from ship to stacking location
(1) Total (sec) for import operation during the simulation run.
(2) Unit (sec/box)

Performance metric of container terminal is a numbers game with all important throughput figures often featuring as benchmarks. There is not a single holistic benchmark that can be applied asses a container terminal performance. Therefore, carefully identifying characteristics of the handling activity should lead to more accurate indicators and targets. This paper focuses on terminal productivity issue that covers all issues driven by the detail performance of container handling equipment used in both parallel and perpendicular layout. We present the performance criteria that are used to evaluate advantage and disadvantage between those two layouts in Table 1. Aside from commonly used metrics, we add new metrics that will show the difference of both layouts in terms of equipment utilization that will closely relate to the performance of local system in respect to global productivity of container terminal.

\section{COMPUTER SIMULATION SETUP}

Object oriented simulation models have been used intensively to understand the behaviour and test different strategies in the container terminal systems, e.g. see [5],[6],[7],[8]. These simulators differ widely in objectives, complexity, and details. Liu et.al. [9] developed a simulation model used to demonstrate the impact of AGV deployment for parallel and perpendicular terminal layout and used multi attribute decision making to assess the performance. The result showed that the use of AGV brings substantial performance effect to container terminal and has different effect considering different terminal layout. Taner et.al. [10] obtained similar result by examining the effect of dispatching rules and resource allocation strategies specifically for perpendicular layout of automated terminal. Both papers showed that each layout format requires a unique combination of cargo handling 
machineries. However, detail performance benchmarks for both layouts were not presented.

In this paper, we focused on building object-oriented simulation model tested on import operation (incoming containers from ship) and see how various arrangements work to get every performance metrics that we desired. The main objective to build the model of sub-systems in the terminal as close to reality considering its motions, inter-relation between equipment and terminal operator logic for stacking strategies. Duration for each run in the constructed simulation model is fixed to 8 hours according to actual work shift of equipment in the container terminal.

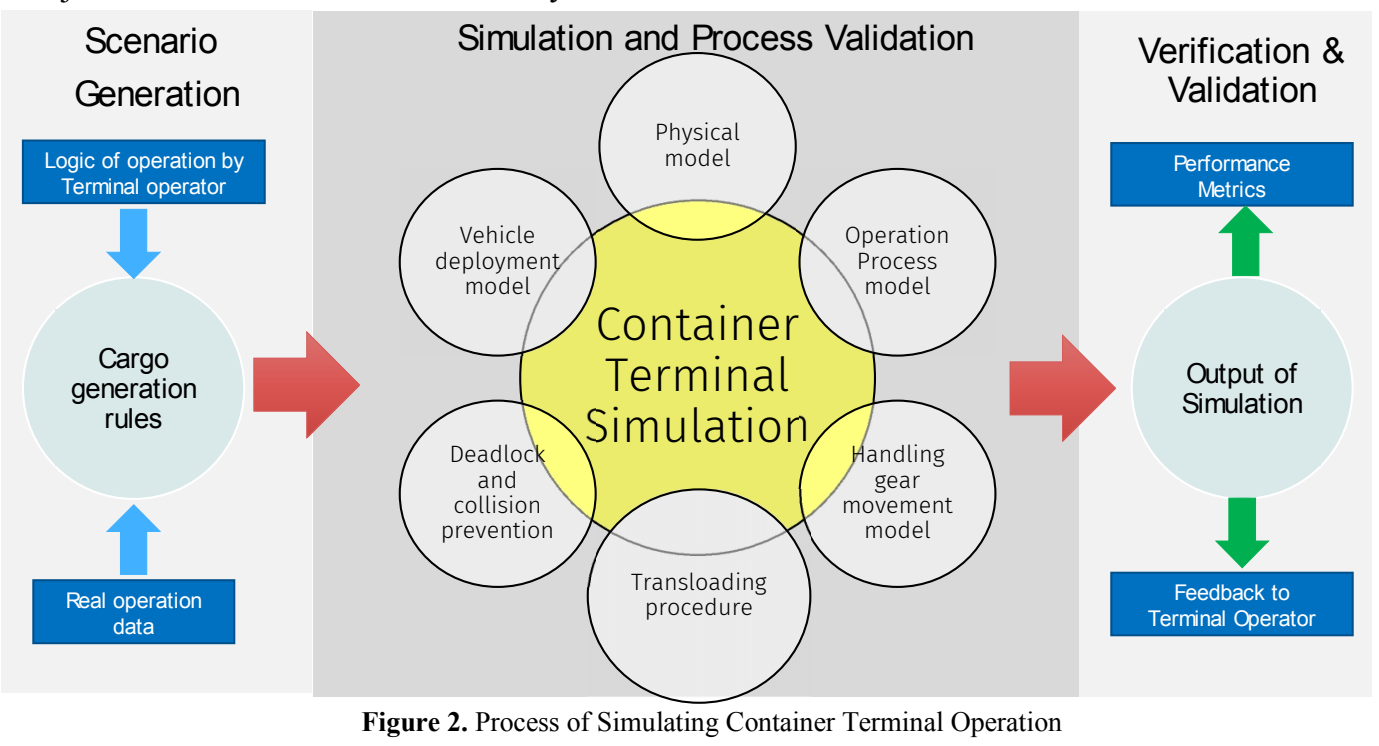

The process flow to build the simulation model is as shown by Figure 2. To depict the reality, input data of the import containers is generated in advance based on actual unloading sequences from ship. Pseudo-random numbers are used to generate loading sequence so that the results for a given scenario can be reproduced. A unique container number, unloading sequence and stacking position are generated using Mersenne Twister algorithm introduced by Matsumoto \& Nishimura, [11] which completely independent of one another.

By iteratively checking flow of input and quality of output data as well as using simulation animation, we were able to detect actions that are illogical to ensure that the simulation model accurately reflects the conceptual model and then fix the computer model. We simulate 5 numbers of replications for each simulation setup, compute the sample variance of the selected estimate, and have determined that the width of the resulting confidence interval is within acceptable limits.

\section{A. 3-Dimensional Model of Terminal Arrangement}

In port operations and management research, virtual modelling has proven to be a powerful tool to design and analyse real world complex situations. Even more important is how to visualize the computer model to what can be called as the terminal digital twin. Constructing 3-Dimensional computer model of container terminal should made us able to make a detail layout, appearance, even the movement of every subsystems in the terminal e.g.; gantry crane system, carrier system, transfer crane system and the connection between those systems. In doing so, we use AutoMod, a general simulation software for discrete event simulation with 3D visualization capability. 3D model for every handling-equipment are constructed by $\mathrm{CAD}$ software, and the equipment's working parameters such as speed, acceleration, stopping distance and hoist/lowering motions are set as individual parameter for discrete event simulation. The value for these motions is gained from work observation at ICCT terminal.

For the general arrangement of the terminal, there are four layout and arrangement models that has been built and simulated as shown by Figure 3. Two models for parallel layout: Parallel_6TC_6Lane (Model A) and Parallel_6TC_3Lane_Landside (Model B) and two models for perpendicular layout: Perpendicular 6TC_6Lane (Model C) and Perpendicular 6TC_3Lane (Model D). The equipment arrangements for both layouts are explained briefly in the following sub-sections.

\section{B. Arrangement of Parallel Type Layout}

For parallel layout, we assumed that two gantry cranes (GC) are serving a container ship at the berth. Model A serves as the basic model to be evaluated against anoter models. For the experimentation purpose, only one ship berth and six container blocks/lanes (24 bays $\mathrm{x} 6$ rows $\mathrm{x}$ 4 tier for each) in the coverage area of the berth will be examined and considered as the basic terminal dimension to be compared with perpendicular layout. In addition, 24 container transfer points (TP) are set on the side of each block. Normally, the export containers are placed closer to the berths and import containers are placed closer to the land side. This setup makes truckchassis transporting import containers travels a longer distance than it should be. For evaluation purpose, two stack location models were built; Model A utilize a default 6 storage block with one operating TC on each block currently used by ICCT. We investigate the possibilities of increasing terminal performance by increasing the number of TC working in a storage block at the same time. Therefore, Model B configured to use only 3 blocks on the land side of the terminal (Figure 3b), as a measure to increase TC productivity. 
The number of truck-chassis deployed in the system move in a counter-clockwise loop between the quay and the stack and the amount were varied from 2 to 12 units for every simulation. The truck-chassis served by the GC and TC based on the first-come-first-served (FCFS) rule. It is also assumed that at most six truck-chassis are allowed to wait in any GC queue at each instant of time. In actual operation, truck-chassis may be allowed to travel up to $30 \mathrm{~km} / \mathrm{h}$. In this paper however, it has a forward speed limitation of $22 \mathrm{~km} / \mathrm{h}$ to be same with AGV maximum speed.

\section{Arrangement of Perpendicular Type Layout}

GC arrangement for perpendicular layout is exactly the same with the one used for parallel layout. The storage area arrangement is configured as follows. Ideally, one storage block will have 4 transfer points (TP) to synchronize container transport from AGV to TC. Considering the layout arrangement of parallel layout, modifications were made to fairly compare both layouts. The first model utilizes 6 storage blocks that use one TC and have only one TP on each (Figure 3c). The second model utilizes 3 storage blocks that use one TC and have two TP on each (Figure 3d). For both model, only 12 bay lengths on each block are active for import container stack out of 24 bays. We also tried to add an innovated concept of yard container transfer that has been introduced in several automated container terminals, e.g. docking station. The key of docking station concept is to provide buffer area where AGVs can unload a container without having to rely and wait on TCs so it can perform the next task without delay.

The AGV arrangement is also mostly similar to truckchassis arrangement for parallel layout. The different is that the AGV is moving freely in the seaside area after catching container from $\mathrm{GC}$ and find the closest path to the transfer point destination attached to a designated block. AGVs do not have to follow specific loop in order to transport import containers. Furthermore, AGVs can travel forwards, in reverse and can overtake each other. The technical parameters and dimension of the working $\mathrm{AGV}$ in this paper follows the parameters used in [12]. It is $14.8 \mathrm{~m}$ in length and $3 \mathrm{~m}$ in width, making it able to transport containers of different length up to 40 '. The maximum forward/reverse speed is $22 \mathrm{~km} / \mathrm{h}$ with stopping distance of $5 \mathrm{~m}$ when facing other AGV passing by, while curve speed is set to be $11 \mathrm{~km} / \mathrm{h}$ at the maximum. AGV is also served by the GC and TC based on the first-come-first-served (FCFS) rule.

\section{Logic of The Simulation}

The developed AutoMod models contain several local systems and collections of entities. The process system defines the model logic that controls how loads are processed in a model. The simulation logics for every local system and synchronization rules between moving entities controls how containers are processed in a model. In this section, we briefly explain the logic used to do make stacking strategies for both layout and logic to conduct traffic management for $\mathrm{AGV}$ in perpendicular layout since those requires special attention.

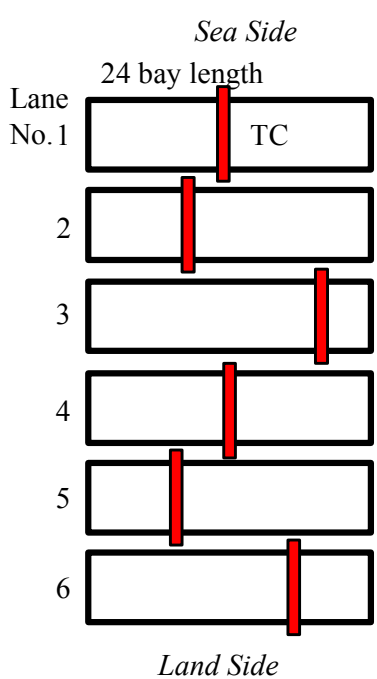

(a) Model A: Parallel 6TC 6Lane

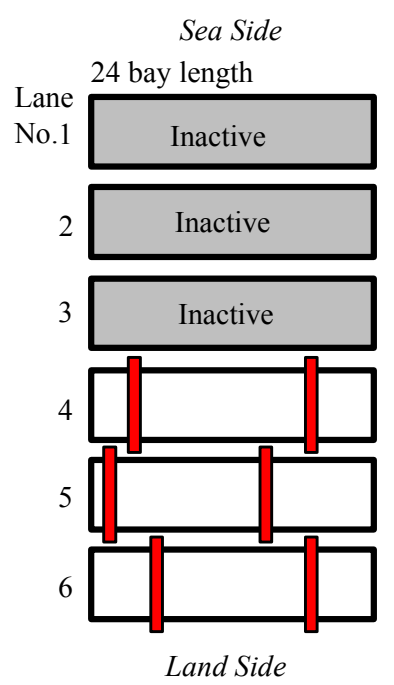

(b) Model B:

Parallel_6TC_3Lane_Landside

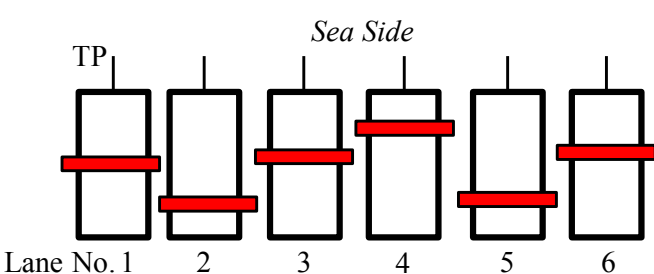

(Only 12 bay length is active for each block)

(c)Model C: Perpendicular_6TC_6Lane

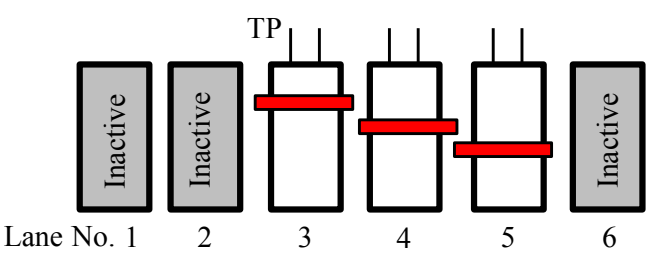

(Only 12 bay length is active for each block)

(d)Model D: Perpendicular_6TC_3Lane

Figure 3. Terminal layout and arrangements tested with simulation 

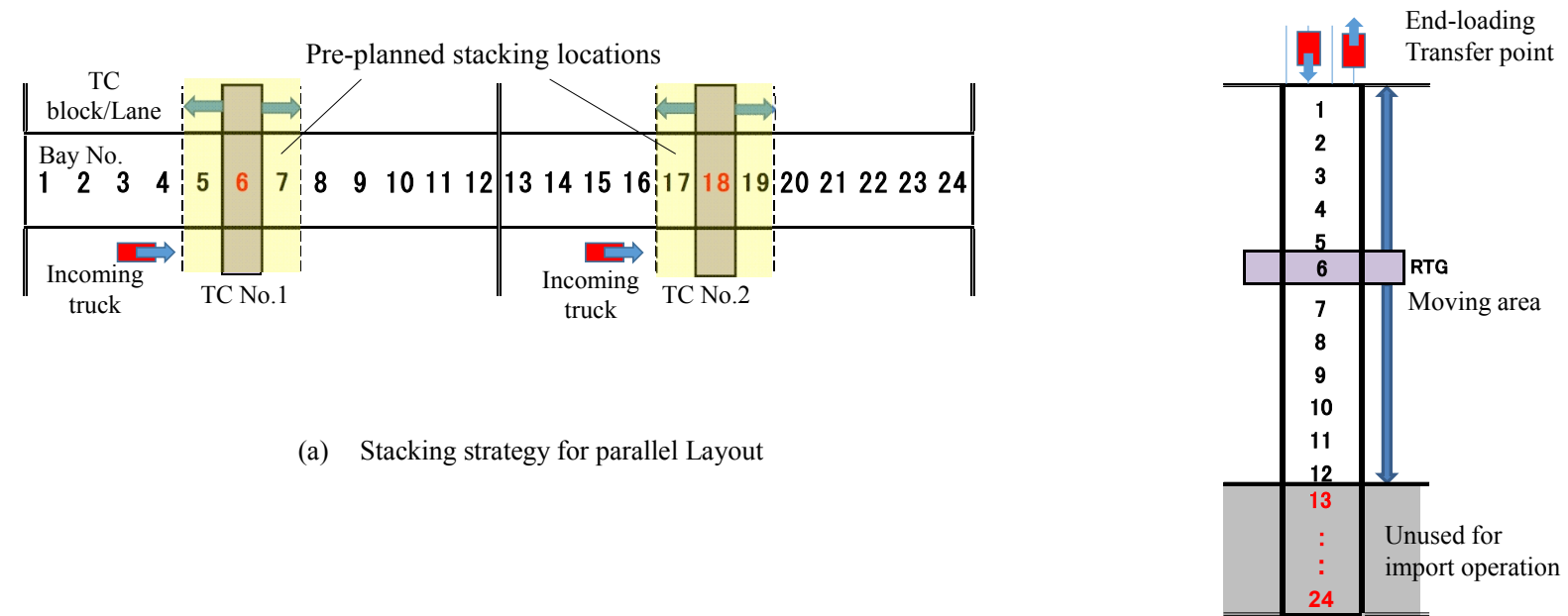

(a) Stacking strategy for parallel Layout

(b) Stacking strategy for Perpendicular Layout

Figure 4. Stacking strategy for both layout to minimize TC movement

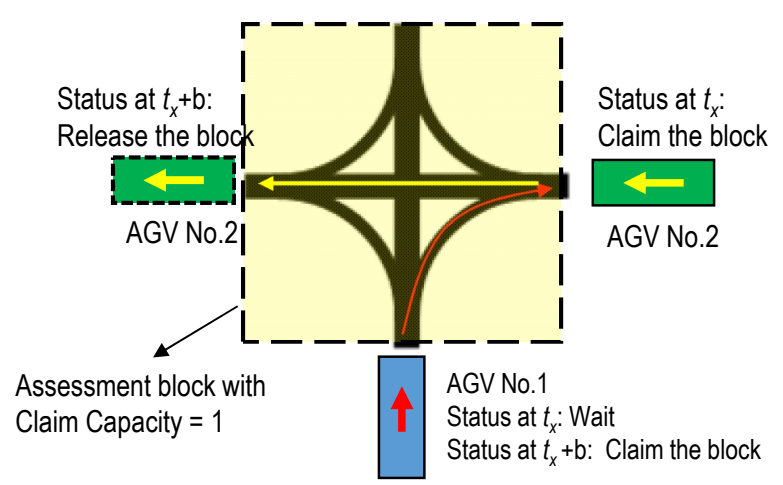

Figure 5. Collision avoidance strategy by implementing maximum claim capacity at intersection.

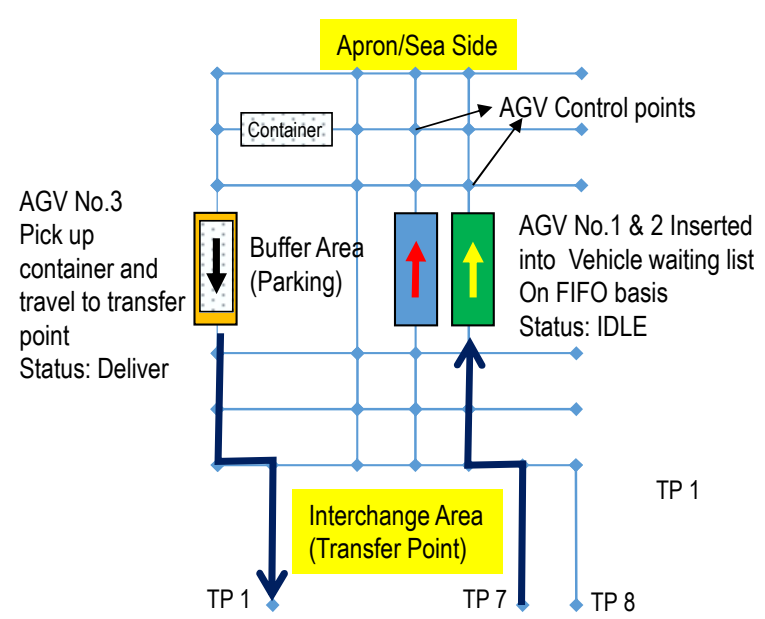

Figure 6. AGV Dispatching rule utilizing buffer area 
(a) Computer Simulation of Parallel Layout

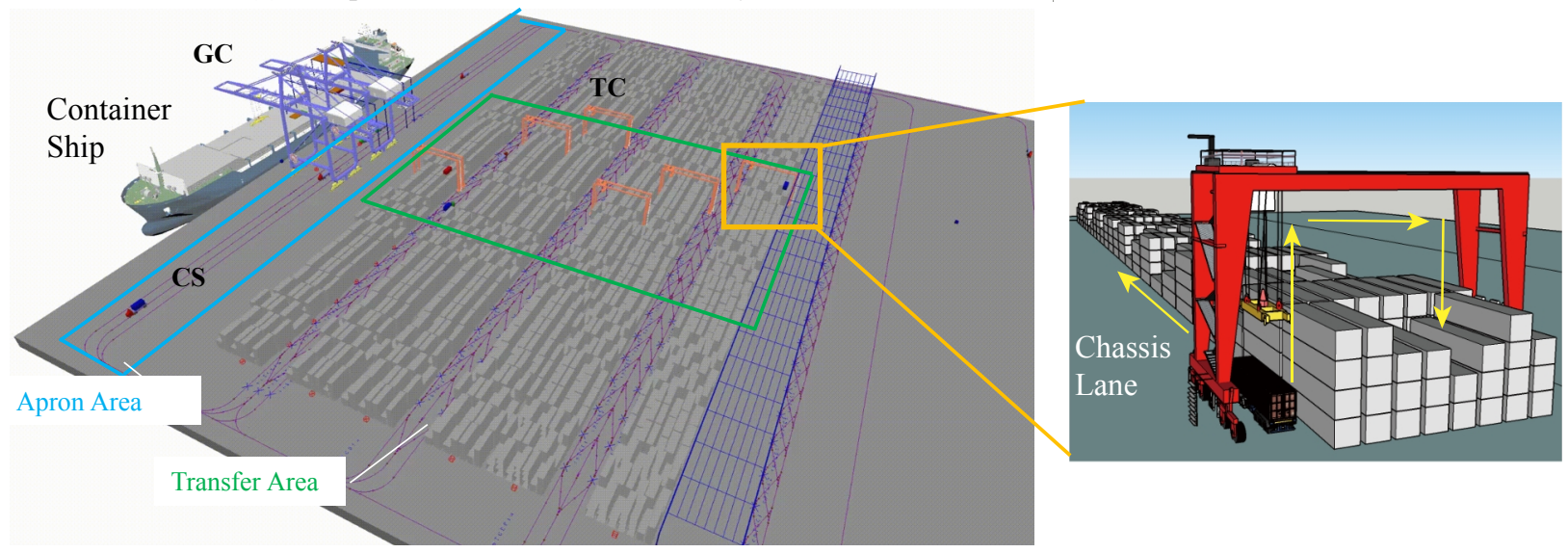

(b) Computer Simulation of Perpendicular Layout
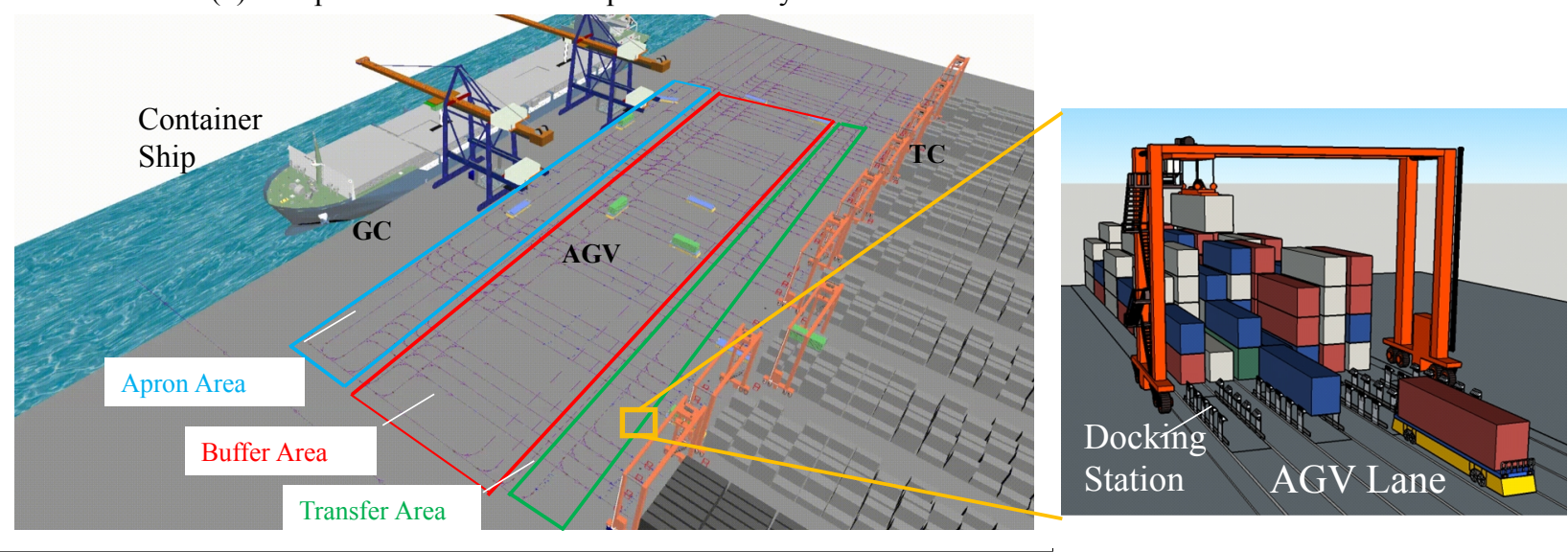

Figure 7. Screenshot of constructed 3D simulation model for both layout in AutoMod

Stacking strategy in storage yard for parallel and perpendicular layout is different, showing how layout orientations have an impact to stacking strategies to maintain stack efficiency and minimize future rehandling. We mimic the parallel layout stacking strategies performed by stack planner in ICCT where a given area in a block are prepared in advance to store import containers to minimize TC's horizontal motions as shown by Figure 4. On the other hand, stacking location for import containers in perpendicular layout can be any given stack positions within the center any container block length to the end of that block on the quay side.

(Box/Hour/GC)

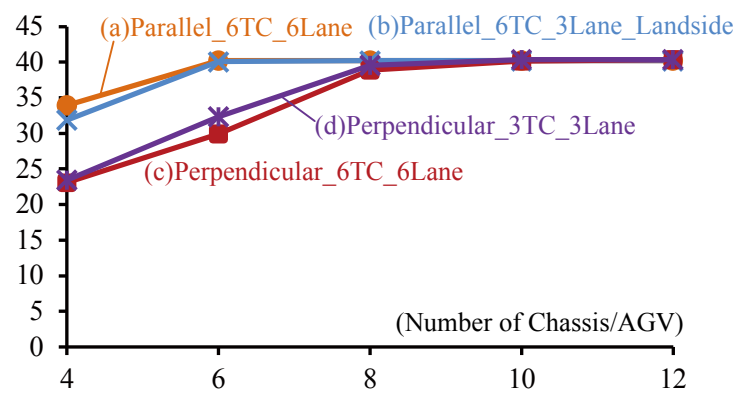

Figure 8. GC Productivity

Furthermore, dispatching rules of AGV is set based on the availability of $\mathrm{AGV}$ and the readiness of container to be transported from the GC. Figure 6 illustrates this
AGV flow in perpendicular layout is somewhat complicated unlike truck-chassis loop flow in parallel layout. Due to the nature of the layout, AGV traffic is inevitable. AGVs may cross-passing each other and deadlock condition might happen. To alleviate this, three traffic management solutions are being implemented e.g.; collision avoidance, dispatching rules and routing. A specific collision avoidance rule based on hierarchical system called semaphores [13], is implemented as a flag in the simulation logic to control the traffic particularly at the intersections as shown by Figure 5. A flag is raised by an AGV that claiming a specific assessment block and this flag is notified to other AGV's in the area. (Sec/Box)

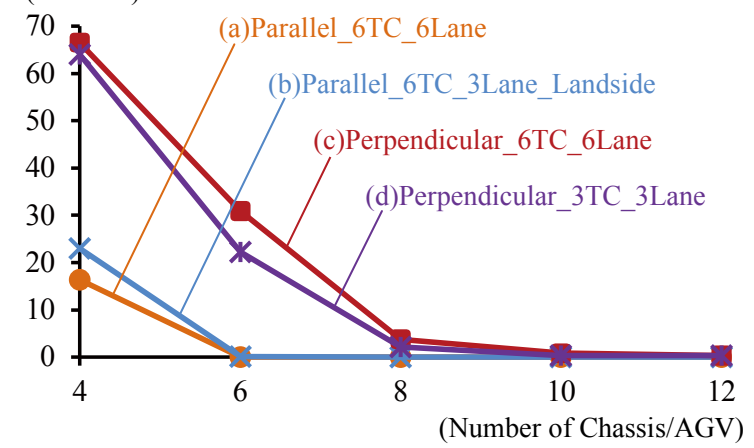

Figure 9. Unit GC waiting time

dispatching concept. An empty AGV will change status from "work" to "idle" and travel to buffer area in between the quay and stacking yard. This buffer area has 
several slots and only one empty AGV can claim the slot at an instant time. Then the AGV that has arrived at the buffer area will be placed at the end of vehicle waiting list to be notified when any container is ready to be picked up.

Finally, routing of $\mathrm{AGV}$ is done by placing traffic control points in the carrier system sub system. AGV Paths are drawn using different primitives (straight sections, curves, stopping) and control points for interaction are snapped on them. Each control point can only be assigned to one path [14]. After confirming a shortest route to destination using Dijkstra's algorithm [15], any moving AGV have to claim a control point on every path along the route and release it after passing the path. Every control point has claiming capacity of 1(one), means that once it has been claimed by an AGV, the second AGV tries to claim the same control point has to wait on its current position until the control point has been released by the first $\mathrm{AGV}$, or find another control point to be claimed and re-routes from its original route. These procedures evolve over time as the simulation runs to alleviate deadlock condition. The computer simulation for both container terminal layout and arrangement is made by implementing all these simulation logics, as depicted by Figure 7 .

\section{RESULTS AND DISCUSSIONS}

In this chapter, productivity measures of both evaluated terminal can be evaluated using the performance metrics introduced in this papers. There are six selected performance metric used to benchmark the existing, nonautomated terminal arrangement (Model A) with three other proposed arrangement models.

\section{A. Comparing Gantry Crane Performance Metrics}

GC productivity for all evaluated models is shown in

Figure 8. This is main performance metric showing the amount of container handled by one GC per one hour. Effort to reach optimum GC productivity at 40 box $/ \mathrm{hr}$ is considered as highly important since it will directly correlate to container ship's berthing time. Obtaining GC maximum productivity will also means that GC doesn't have to wait for carrier system arrival, and there is no waiting time of GC. Therefore it is safe to assume that GC waiting time as shown in Figure 9 is directly related to the GC productivity.

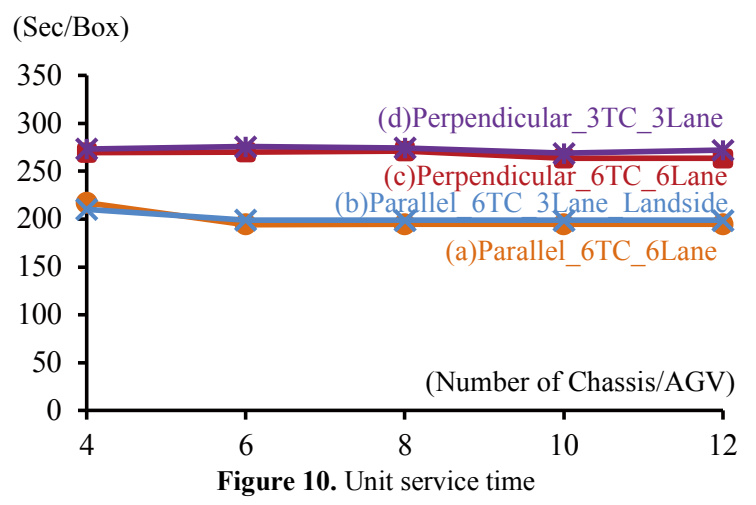

In terms of the trade-off between GC productivity and carrier system's (CS) utilization, Model A and B reached maximum productivity by utilizing $6 \mathrm{CS}$, while Model $\mathrm{C}$ and D needs $8 \mathrm{CS}$. This result inferred that Parallel layout is more efficient than Perpendicular layout from carrier system installation cost perspective. Even in case of using docking station, the stations have a limited capacity. Having to wait for TC to pick up container docked at any station, incoming AGV with container on it will have to wait until docking stations are available. However, due to less amount of TC is required for Model D compare to the other model, cost advantage from lower number of TC exceeds disadvantage of GC productivity, then Model $\mathrm{D}$ case might become adequate choice when GC maximum productivity is not become the main target of operation.

\section{B. Comparing Unit Service Time for Single Container}

Unit service time is shown by Figure 10. As a concept of measuring total performance, unit service time is described as the total duration that is needed to handle one container from ship stowage to designated stacking location in the storage yard. So, unit service time shows the whole utilization of cargo handling equipment required to transport a single container. It also shows efficiency of each handling case. For instance, unit service time of Model B is a bit higher than model A in any number of truck-chassis arrangement. This is because stacking lane is unevenly distributed to the land side in Model B and truck-chassis need to run long distance on average compare to Model A. Consequently, unit service time of parallel layout models (Model A and $\mathrm{B})$ is generally lower than that of perpendicular models (Model C and D).

This result shows that parallel layout is more effective than perpendicular case from the speed of container handling perspective. However, this parallel model's unit service time is shorter than required to maximize GC handling productivity. A fair analysis can be drawn by examining the simulation animation. When the deployed number of carrier system is $8, \mathrm{GC}$ handling productivity for Model A, B and C is 40box/hour/GC and there is no $\mathrm{GC}$ waiting time, but, unit service time of Model $\mathrm{C}$ is about 270 second. On the other hand, unit service time of Model A and B is about 200 second. This means that the unit service time for perpendicular layout using Model A is enough to maximize GC handling while unit service time for parallel layout is too shorter than that is needed.

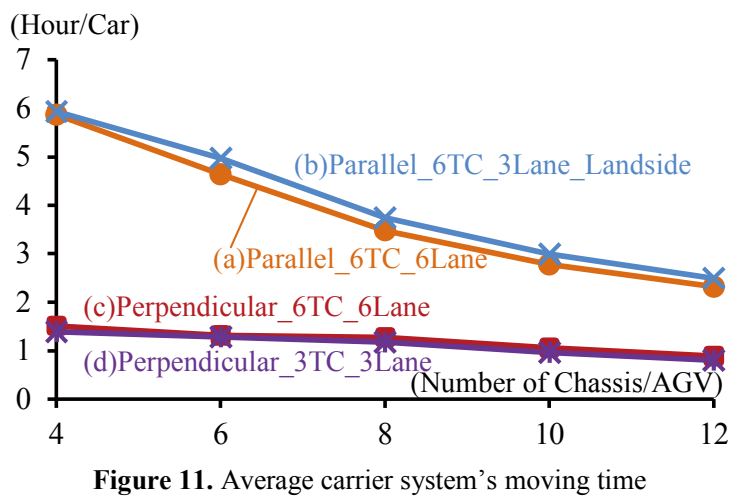




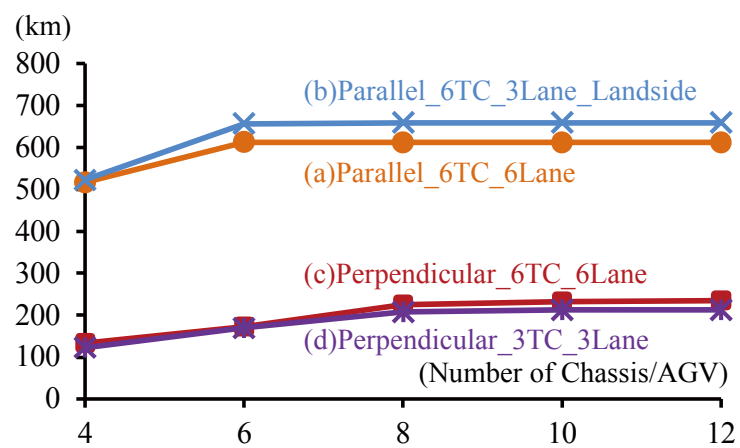

Figure 11. Total distance covered by carrier systems for both layouts

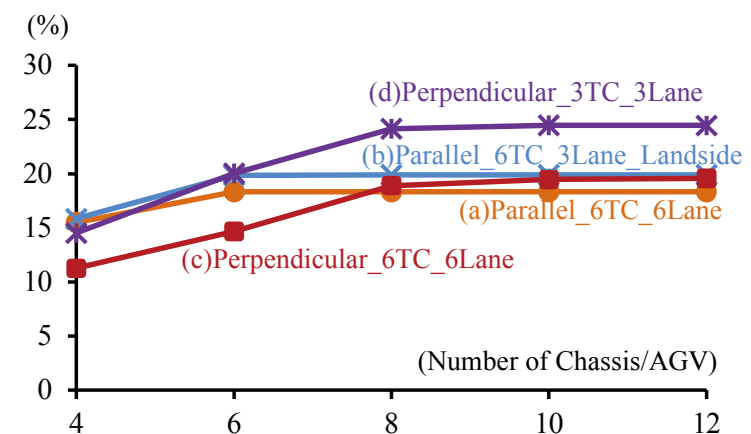

Figure 12. Average percentage time in motion for TC in both layout

\section{Comparing Carrier System's Performance Metrics}

Average carrier system's moving time is shown by Figure 11. Using this metric, carrier systems expected energy consumption can be evaluated. Furthermore, Chassis and AGV utilization rate can be confirmed. If this moving duration is low, then that means that carrier system is idling or stop for long duration. From Figure 8, it can be concluded that truck-chassis moving duration of each parallel layout models is longer than AGV moving duration of perpendicular layout models. This is mainly due to the difference in carrier system moving path that comes as the consequences of layout orientation and yard transfer point locations. Shorter path of $A G V$ is one of advantage of perpendicular layout. However, low moving time is also means that AGV stop and wait for long time and AGV usage rate in perpendicular case is lower than truck-chassis usage rate in parallel case. Incidentally, Chassis energy consumption rate and AGV energy consumption rate that based on moving duration is different. Therefore, it is impossible comparing truckchassis and AGV energy consumption only by measuring the moving time duration.

In addition, truck-chassis moving duration in parallel layout models is decrease with the increase number of truck-chassis being deployed. This is not the case for perpendicular layout models. A GV moving duration does not decrease with the increase in number of AGVs as much as the parallel model cases. Carrier system total distance for all models is shown by Figure 12. It show the merit of perpendicular layout in terms of reducing horizontal distances that have to be covered by carrier system for various setups. The total covered distances are saturated after utilizing some number of carrier systems in the system. Correlating this finding to Figure 5 , it is safe to conclude that carrier system's deployment above a certain level would not be necessary since GC productivity would not be increase further. In addition, this metric can be used to calculate carrier system energy consumption in pair with Figure 11.

The last metric explained in this paper is the average $\mathrm{TC}$ 's percent time in motion shown by Figure 12. While many researcher in container terminal operation focusing only on GC and CS metrics, most of us neglect to consider the importance of $\mathrm{TC}$ metrics in measuring the total performance of terminal system. While it is of the same importance, up until this manuscript is written, there is no measurement technique available to measures TC metrics due to lack of method in correctly collect the $\mathrm{TC}$ metric in simulation models. We were able to collect

TC performance metric due to high-level detail in modeling the TC movement, from retrieving the container on top of any carrier system, traveling on its four-wheels up to stacking a container using its trolley.

We consider the TC metric as the percentage of time where the $\mathrm{TC}$ is in motion. In other word, TC utilization is hypothetically most effective when it's working duration is larger than its idle time waiting for a container to handle. This metric is unique because it covers the duration percentage of each TC move in any mode of TC work in its moving sequence e.g. horizontal movement to retrieve, picking-up container from AGV by trolley, horizontal movement to delivery and container set-down by trolley at designated stack location. In other word, this metric shows how $\mathrm{TC}$ is affected by layout orientation and equipment arrangement. Therefore, utilization rate of $\mathrm{TC}$ is able to evaluated by refer to this metric.

Simulation results show us that $\mathrm{TC}$ percent time in motion for Model B is slightly higher than that of Model A. This implies that the TC utilization rate for parallel layout can be increased by using two TC operates at the same container block/lane. This finding is important to be disclosed to terminal operator searching a measure to increase their productivity by $\mathrm{TC}$ dispatching strategy. On the other hand, TC percent time in motion for Model $D$ is higher than that of Model C. This finding show that the efficiency of perpendicular layout utilizing smaller amount of container block/lane will be lower, even when the number of active transfer points and docking stations is increased.

B y pairing Figure 7 and Figure 12 we can draw a general conclusion regarding TC utilization rate. Note that maximum GC productivity can be reached by deploying 6 prime-movers for Model A, B (parallel layout). In this case, TC motions can be inferred as the amount of energy consumed by TC. To reach the same amount of productivity, note that the single $T C$ in perpendicular layout is expected to consume more energy due to its higher percentage in motion compare to a single TC in parallel layout. Again, due to the nature of the layout shown by Figure 1 b and Figure $2 \mathrm{c}$, a $\mathrm{TC}$ in perpendicular layout has to go back to the end side of a container block/lane to retrieve container from the docking station. This retrieval operation that consumes energy is alleviated in case of parallel layout because a stack area that closes to one another can be prepared in advance to minimize TC horizontal movement as shown by Figure 4 . On different point of view, TC percent time 
in motion can also be cross-interpreted as TC utilization rate. In this sense, one can point out that TC usage rate for parallel models may be lesser than that of perpendicular models in low amount of prime-movers case. Amount of TC and performance is a trade-off in operation and we might be able to be decrease the number of TC being utilizes with an awareness to keep GC under its maximum productivity.

\section{CONCLUSION}

Adoption of dramatic innovation in the handling systems such as automation technology in container terminal is a difficult task for terminal having softer annual throughput compared to major terminal players. Hard infrastructure of the automated terminal might requires a major and significant change to container terminal layout. Evaluation items need to be set for the operation planning and design of the container terminal, especially for the functional evaluation of the newly adopted technology in comparison to the current technology. In this paper, we investigated, through developing benchmark for measuring performance metrics as well as simulation models, the impact of layout orientations and terminal arrangements on overall performance of container terminal systems.

An existing layout and arrangement is compared with three conceptual models incorporating stacking allocation strategy, vehicle dispatching strategy and the use of different non-automated and automated cargo handling equipment. We showed the merit of perpendicular layout in automated terminal in comparison to parallel layout in non-automated terminal in terms of minimizing horizontal transport as well as its disadvantage in terms of heavy burden given to yard transfer crane. Based on the findings, a research agenda can be made in order to examine a way to reduce the burden of yard transfer crane in automated container terminal utilizing perpendicular layout.

Apart from the automation technology, yard layout has inevitable effects to the terminal performance due to effort that has to be made by handling equipment to reach optimum productivity. Our result also shows that arrangement of two transfer crane serving at the same parallel storage block show a great promise in increasing existing container terminal productivity

\section{REFERENCES}

[1] K.H. Kim and H. Lee, "Container terminal operation: current trends and future challenges" in Handbook of Ocean Container Transport Logistics, International Series in Operations Research \& Management Science 220, 2015, pp.43-73.

[2] H. Cederqvist,. "Container terminal yard automation", PEMA Information paper, Port Equipment Manufacturers Association, London, United Kingdom, 2012

[3] K. Bichou, Port Operations, Planning and Logistics. New York: Informa Law from Routledge, 2013.

[4] P. Hangga and T. Shinoda, "Motion-based energy analysis methodology for hybrid straddle carrier towards eco-friendly container handling system", Journal of the Eastern Asia Society for Transportation Studies vol.11, pp.2412-2431, 2015.

[5] Y. Hayuth, , M.A. Pollatschek, and Y. Roll, "Building a port simulator". Simulation, vol.63 (3), pp. 179-189, 1994.

[6] W. Y. Yun, and Y. S. Choi, "A simulation model for containerterminal operation analysis using an object-oriented approach", Int.Journal of Prod. Economics, vol.59, pp. 221-230, 1999.
[7] E. Kozan, "Comparison of analytical and simulation planning models of seaport container terminals". Transportation Planning and Technology, vol.20, pp. 235-248, 1997.

[8] M. Bielli, A. Boulmakoul, and M. Rida, "Object oriented model for container terminal distributed simulation". European Journal of Operation Research, vol.175, pp. 1731-1751, 2006.

[9] Liu, C.I., Jula, H. Vukadinovic, K. Ioannou, P. (2004). Automated guided vehicle system for two container yard layouts. Transportation Research Part C, 12, pp. 349-368.

[10] M.E. Taner, O. Kulak, and M.U. Koyuncuoğlu, "Layout analysis affecting strategic decisions in artificial container terminals". Computers \& Industrial Engineering, vol.75, pp.1-12, 2014.

[11] M. Matsumoto and T. Nishimura, "Mersenne Twister: A 623 dimensionally equidistributed uniform pseudo-random number generator", ACM Transactions on Modeling and Computer Simulation, vol.8-1, pp.3-30, 1998.

[12] J. Široký, "Automatic Transhipment Systems for Container Transport in Terminals". Perner's Contacts, vol.6(3), pp.145154, 2011.

[13] J. J. M. Evers and S. A. J. Koppers, "Automated guided vehicle traffic control at a container terminal", Transportation Research Part A: Policy and Practice, vol. 30(1), pp. 21-34, 1996.

[14] K. Gutenschwager, A. Radtke, S. V"olker, and G. Zeller, "The Shortest Path: Comparison of Different Approaches and Implementations for the Automatic Routing of Vehicles", Proceedings of the 2012 Winter Simulation Conference, pp.3312-3323, 2012

[15] E.W. Dijkstra, "A note on two problems in connexion with graphs", Numerische Mathematik vol.1, pp.269-271, 1959. 